\title{
Differential recognition of the right vs. left halves of human faces
}

\author{
DARLENE F. KENNEDY, CARMELLA C. SCANNAPIECO, \\ SUSAN M. MILLS, and W. J. CARR \\ Beaver College, Glenside, Pennsylvania
}

\begin{abstract}
In an earlier facial recognition study, subjects inspected 6 full faces and, 1 week later, tried to identify those 6 from among 12 faces that were presented as full faces, left halves only, or right halves only. Fewer errors were committed by subjects who viewed faces that were left half only than by subjects who viewed faces that were right half only. Such a finding could stem from differences in the amount of information contained in the left versus right halves of faces or from differences in the amount of information extracted by the subjects. The present experiment supports the latter explanation by showing the same advantage of left half over right half even when the faces used were mirror images of the faces used in the earlier experiment. In both experiments, fewer errors were committed by subjects who viewed full faces during testing than by subjects who viewed partial faces.
\end{abstract}

Studies of facial recognition indicate that humans have a large capacity for encoding and recognizing normally oriented faces (Freedman \& Haber, 1974; Hochberg \& Galper, 1967). Within each face, the cues provided by individual features may be an important factor affecting recognition. In facial recognition, as in the recognition of many objects, not all attributes of the stimulus receive the same amounts of attention. Some elements of a face may be more important than others in the recognition of that face. For example, Goldstein and Mackenberg (1966) and Seamon, Stolz, Bass, and Chatinover (1978) found that eyes provided more information for the recognition of a previously seen face than did other features. Goldstein and Mackenberg also reported that the upper half of a face provided more information or cues to allow the recognition of a previously seen full face than did the lower half. They did not compare the left versus right halves of faces, perhaps because they thought of the two sides as being functionally equivalent.

In an earlier facial recognition test (Kennedy, Beard, \& Carr, 1982), subjects inspected 6 full faces for $5 \mathrm{~min}$ and, 1 week later, tried to identify those 6 from among 12 faces that were presented as full faces, left half only, or right half only. As might be expected, the subjects who viewed full faces during testing committed the fewest errors, but those who viewed the left half also committed fewer errors than did those who viewed the right half. The latter finding might stem from a difference in the amount of information actually contained in the left versus right halves of the faces or, more likely, from a difference in the amount of information extracted via central processing from each half during inspection (Gilbert \& Bakan, 1973). The present experiment tested these two

The authors' mailing address is: Department of Psychology, Beaver College, Glenside, PA 19038. hypotheses by repeating the earlier experiment in all respects, except that the faces used in the present experiment were mirror images of those used earlier by Kennedy et al. (1982). In both experiments, the left half of a face was defined as the side a person would call his/her own left side.

\section{METHOD}

\section{Subjects}

Ninety-nine college undergraduates viewed six full faces during the inspection phase. The subjects were then assigned randomly to one of three groups ( $\mathrm{N}=30-36$ per group), differing only with respect to the aspect of the faces presented 1 week later during a recognition test (i.e., full face, left half of face, or right half).

\section{Materials}

The faces used in the present experiment were the same as those prepared by Cohen and Carr (1975), and used by Kennedy et al. (1982). Twelve photographs of adult male faces (frontal view) were selected randomly from a large pool of photographs. The photographs were of males between the ages of 22 and 40 years; the taces displayed no emotion and possessed no unusual features (e.g., scars, tiards, eyeglasses, etc.). The 12 photographs were converted to standardized line drawings via the Identi-Kit (Smith-Wesson Law Enforcement Group, Santa Ana, CA).

For the present experiment, the faces were photographed in a reversed or mirror-image orientation, so that what was present on the right half of a normally oriented face would be present on the left half of the mirrorimage face. Copies were made of the 6 full faces arranged on a single sheet of paper (the inspection sheet), and copies were made of all 12 faces on each version of test sheet. Test sheets for the experiment were of three types: full face, left half of a face, or right half of a face.

\section{Procedure}

During the inspection phase of the experiment, each subject viewed an inspection sheet containing the six full faces for $5 \mathrm{~min}$ and was instructed to remember the faces but not to discuss the experiment with others. One week later, approximately one-third of the subjects were presented with one of the three types of test sheet (full face, left half, or right half of a face) containing 12 faces. They were asked to identify the 6 faces that had been seen during the previous inspection, and to guess when necessary. No time limit was imposed on the recognition test. 


\section{RESULTS}

During the recognition test, the 99 subjects were able to identify the six faces seen 1 week earlier rather well. Only 134 of 1,188 possible errors were committed, an overall error rate of $11.3 \%$. The error rate for the subjects who viewed full faces, left halves, and right halves were $7.8 \%, 8.6 \%$, and $16.6 \%$, respectively.

Overall, the three groups differed reliably with respect to the number of errors committed [Kruskal-Wallis oneway ANOVA, $\mathrm{H}(2)=7.58, \mathrm{p}<.05]$. Moreover, the subjects who viewed full faces during the recognition test committed reliably fewer errors than did those who viewed the right halves (Mann-Whitney $\mathrm{U}$ test, $\mathrm{z}=2.68$, $\mathrm{p}<.05$ ). But the subjects who viewed full faces did not differ reliably from those who viewed the left halves $(\mathrm{z}$ $=0.74)$. More importantly, the subjects who viewed the left halves committed reliably fewer errors than did those who viewed the right halves $(\mathrm{z}=2.32, \mathrm{p}<.05)$.

\section{DISCUSSION}

Kennedy et al. (1982) permitted subjects to inspect 6 full faces for $5 \mathrm{~min}$ and, 1 week later, asked them to identify the 6 faces that had been seen earlier from among 12 faces. They found that subjects who viewed only the left halves of the 12 faces committed fewer errors than those who viewed only the right halves. The present experiment reveals the same effect even though the faces used in this experiment were the mirror images of the 12 faces used by Kennedy et al. (1982). Therefore, we infer that the effect stems from a difference in the amount of information extracted via central processing from each half during inspection, and not from a difference in the amount of information actually contained in each half.

Earlier studies indicate that the right half of the human face looks more like the whole face than does the left half (Gilbert \& Bakan, 1973; Sackheim, Gur, \& Saucey, 1978), a finding that seems incongruent with that reported here. But the incongruity may stem from differences in methodology. In the earlier studies, the faces were inspected for the first time either during the test or just before it, and the subjects were asked to make judgments about the faces, for example, the degree of similarity or emotional intensity. In the present test, the subjects were asked to identify the 6 faces they had inspected 1 week earlier from among 12 faces. Our interpretation of the incongruity is in accord with a report by Patterson and Bradshaw (1975), who theorized that recognition tasks that involve easy discriminations and short-term memory confer an advantage to the left visual field, whereas tasks that involve difficult discriminations and long-term memory confer an advantage to the right visual field.

\section{REFERENCES}

CoHen, M. E., \& CARR, W. J. (1975). Facial recognition and the von Restorff effect. Bulletin of the Psychonomic Society, 6, 383-384.

Freedman, J., \& HABER, R. N. (1974). One reason we rarely forget a face. Bulletin of the Psychonomic Society, 3, 107-109.

Gilbert, C., \& BAKAN, P. (1973). Visual asymmetry in perception of faces. Neuropsychologia, 11, 335-362.

Goldstein, A. G., \& Mackenberg, E. J. (1966). Recognition of human faces from isolated facial features: A developmental study. Psychonomic Science, 6, 149-150.

HochberG, J., \& GALPER, R. E. (1967). Recognition of faces: I. An exploratory study. Psychonomic Science, 9, 619-620.

Kennedy, D., BeARD, D., \& CARR, W. J. (1982). Differential recognition of the left vs. the right side of human faces. Bulletin of the Psychonomic Society, 20, 72-73.

Patterson, K., \& Bradshaw, J. L. (1975). Differential hemispheric mediation of nonverbal visual stimuli. Journal of Experimental Psychology: Human Perception and Performance, 1, 246-252.

Sackheim, H. A., Gur, R. C., \& Saucy, M. C. (1978). Emotions are expressed more intensely on the left side of the face. Science, 202, 434-435.

Seamon, J. G., Stolz, J. A., Bass, D. H., \& Chatinover, A. I. (1978). Recognition of facial features in immediate memory. Bulletin of the Psychonomic Society, 12, 231-234.

(Manuscript received for publication December 16, 1984.) 